\title{
Uusioersily
}

\section{Components of a community model of dementia palliative care}

Fox, S., O'Connor, N., Drennan, J., Guerin, S., Kernohan, W. G., Murphy, A., \& Timmons, S. (2020).

Components of a community model of dementia palliative care. Journal of Integrated Care, 28(4), $349-364$.

https://doi.org/10.1108/JICA-02-2020-0013

Link to publication record in Ulster University Research Portal

\section{Published in:}

Journal of Integrated Care

Publication Status:

Published (in print/issue): 30/06/2020

DOI:

https://doi.org/10.1108/JICA-02-2020-0013

\section{Document Version}

Author Accepted version

\section{General rights}

Copyright for the publications made accessible via Ulster University's Research Portal is retained by the author(s) and / or other copyright owners and it is a condition of accessing these publications that users recognise and abide by the legal requirements associated with these rights.

\section{Take down policy}

The Research Portal is Ulster University's institutional repository that provides access to Ulster's research outputs. Every effort has been made to ensure that content in the Research Portal does not infringe any person's rights, or applicable UK laws. If you discover content in the Research Portal that you believe breaches copyright or violates any law, please contact pure-support@ulster.ac.uk. 


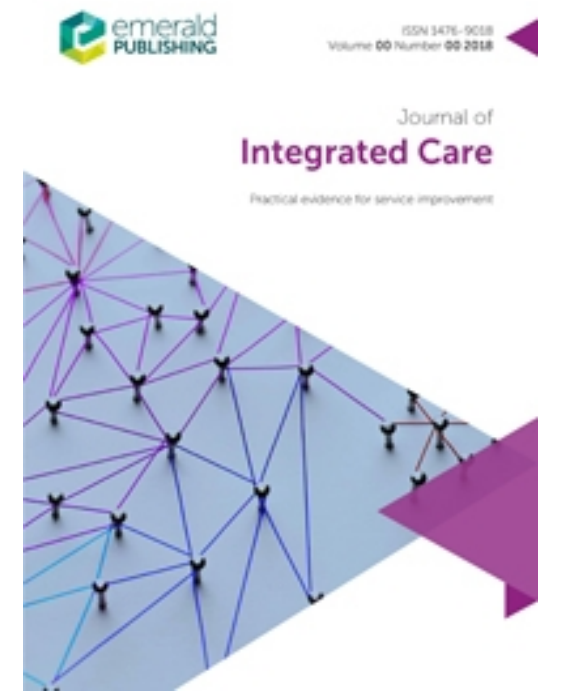

Components of community model of dementia palliative care

\begin{tabular}{|r|l|}
\hline Journal: & Journal of Integrated Care \\
\hline Manuscript ID & JICA-02-2020-0013.R2 \\
\hline Manuscript Type: & Article \\
\hline Keywords: & dementia, palliative care, integrated care model, end of life \\
\hline \multicolumn{2}{|c}{} \\
\hline
\end{tabular}

SCHOLARONE $^{\text {TM }}$

Manuscripts 
1 Title: Components of community model of dementia palliative care

\section{Abstract}

3 Purpose: The Model for Dementia Palliative Care Project will develop a service-delivery

4 model for community-based dementia palliative care. Many countries provide dementia

5 palliative care services, albeit with considerable variability within these. However, little is

6 known about what service providers consider to be the most important components of a

7 dementia palliative care model. This study aimed to address this knowledge gap.

8 Design/methodology/approach: An exploratory design using a survey method was used, as

9 an initial phase of the wider project. A web-based survey was developed, piloted $(n=5)$,

10 revised, and distributed within five healthcare jurisdictions: the Republic of Ireland, Northern

11 Ireland, England, Scotland, and Wales. The target population was health and social care

12 professionals, policy-makers, and academics interested in dementia and palliative care.

13 Content analysis of open-ended questions identified common themes; descriptive statistics 14 were applied to the closed-ended questions.

15 Findings: Overall, N=112 complete surveys were received. Key care principles incorporated 16 the philosophies of palliative care and dementia care; many described 'holistic' and 'person17 centred care' as core. Important individual service components were support for carers, 18 advanced care planning, information, education and training, activities for 'meaningful living', comprehensive disease management, coordinated case management, and linking with community health services and social activities. Barriers included poor availability and organisation of healthcare services, stigma, misconceptions around dementia prognosis, insufficient advanced care planning, and dementia-related challenges to care. Facilitators included education, carer support, and therapeutic relationships.

Originality/value: This study, as part of the larger project, will directly inform the development of a novel service delivery Model of Dementia Palliative Care for Ireland. The results can also inform service planning and design in other countries.

Keywords: dementia; palliative care; integrated care model

Author classification: Research paper

\section{Introduction}

31 Dementia is recognised internationally as one of the most significant healthcare challenges. Worldwide, around 50 million people have dementia, and there are nearly 10 million new 
cases every year (World Health Organisation, 2019). Dementia is a degenerative, life-limiting illness. The average survival time from dementia diagnosis varies greatly depending on gender, dementia sub-type, age and severity of symptoms at diagnosis; however, one systematic review found that the average survival time is between 1.1-8.5 years (Brodaty et alet al., 2012). A more recent study of memory clinic patients, including those with young onset dementia, found a median survival time post-diagnosis of just 6 years (RhodiusMeester et alet al., 2019). As there are no current curative treatments, all treatment is essentially palliative, and there is an imperative for research to improve the palliative care of people with dementia (PwD). PwD and their carers have been shown to have palliative care needs equal to those of cancer patients (Dempsey et alet al., 2015). However, the palliative care needs of PwD may be complicated by cognitive impairment, communication difficulties and responsive behaviours; presenting unique challenges to palliative care (Dening et al., $\bar{r}$ Sampson and De Vries, 2019). Qualitative research has shown that while in many ways the basic palliative care needs of people are the same regardless of illness, families' experiences differ, for example families report extra stress when their dying loved one cannot be involved in decision making about their own end of life care (Fox et alet al., 2017). Although some national dementia strategies include a focus on palliative care, such as Ireland, England, Northern Ireland, and Sweden, and Scotland, many do not (Nakanishi et alet al., 2015).

The principles of palliative care and of dementia care are innately complementary. Personcentred care (Kitwood, 2011) is at the heart of good dementia care, and arguably (although not always explicitly stated) also palliative care. Both emphasise a holistic approach, recognising the importance of non-physical symptoms, and of quality rather than duration of life. Both emphasise the patient-family unit, and espouse the importance of multi-way communication and shared decision making, and of future care planning. A useful framework for optimal dementia palliative care has been agreed (Van der Steen, 2014), including, in addition to the above, continuity of care, recognition of dying, avoiding burdensome/futile treatments, and health and social care professional (HSCP) education. Worldwide, there remains a significant need to expand the research base for palliative and end-of-life care, in recognition of emerging global priorities, moving beyond cancer to examine diseases such as dementia (Dening, 2016). Within Europe, the Joint Programme-Neurodegenerative Disease (EU JPND, 2014) suggested the following priorities for palliative and end-of-life care research in neurodegenerative disease: needs assessment, the identification of transitions along the pathway, and consideration of effective models across Europe. However, few evidence-based models of dementia palliative care have been published. 
The Model for Dementia Palliative Care Project aims to explore existing models of palliative care for PwD in the community (theoretical models from the literature and actual clinical services), using a programme theory approach to describe key model components (context, focus, resources/input, service activities, outputs), and then translate these into a model relevant to the community. The principal aim is to develop a service delivery model incorporating key activities, and to provide a cost estimate and value to potential users of the model in Ireland. However, the model will be designed to be translatable to other healthcare systems and contexts. Multiple methods are being employed, including an initial exploratory survey to explore key healthcare stakeholders' views on what is a "good" model of palliative care for PwD living in the community.

The guiding research questions of the exploratory survey were:

- What do stakeholders consider should be the key principles of care to inform a model for dementia palliative care?

- What do stakeholders consider to be the most important components or activities of such a model?

- How should such a model be organised?

- What are perceived barriers to such a model, and how might these be overcome?

\section{Methods}

\section{Survey development}

To identify what stakeholders consider 'a posteriori' to be important in a model of dementia palliative care (based on their experience), a de novo survey was developed based on a literature review and the expert opinion of the project advisory group, consisting of academics and clinicians from dementia and palliative care.

The majority of survey questions were open-ended, intended to elicit rich qualitative data. Two closed-ended questions asked respondents to rate the importance of a variety of 'general aspects' and 'specific components' of a model for dementia palliative care (based on components identified in the literature, augmented by suggestions from the advisory group). These prompted respondents to rate aspects/components which they may or may not have considered in their responses to the earlier open-ended questions, using a 4-point Likert scale, with options 'not very important', 'somewhat important', 'very important', and 'essential'. 
100 A paper version was piloted among a convenience sample $(n=5)$. Following feedback, a 101 modified electronic version was created using LimeSurvey@ , and further piloted for usability $102(n=3)$. The survey questions are provided in Table 1.

\section{$\ll<$ INSERT TABLE 1 ABOUT HERE $\gg>$}

\section{Sampling}

105 Surveys were distributed electronically in the Republic of Ireland, Northern Ireland, Scotland, 106 and England and Wales (five countries, with the latter two sharing a healthcare system). The 107 rationale for choosing these was their comparable healthcare systems, and good contextual 108 similarity to Ireland. A purposive and "snowball" sampling methodology was used (Palinkas 109 et al., 2015). Inclusion criteria were HSCPs/managers/policy-makers or academics with 110 expertise in dementia palliative care. The initial purposive recipient pool was informed by 111 national healthcare databases and websites, the knowledge of the research team and their 112 extended networks, in each region, and by direct contact with service leads for dementia and 113 for palliative care in each country. Priority was given to targeting key stakeholders and 114 experts, over final sample size. Initial recipients were asked to forward the email to any 115 contacts they considered relevant, to increase inclusion.

\section{Data analysis}

117 Raw data were imported into Excel and cleaned. Descriptive statistics and graphs illustrated 118 the quantitative data. The open-ended questions provided abundant qualitative data, which 119 was analysed using Thematic Content Analysis (-following Braun and Clarke's 200ㅁg 120 framework). The first stage looked for key themes within individual survey questions, using 121 an inductive and descriptive approach; subsequent stages of analysis considered themes 122 across the full dataset deductively, driven by the guiding research questions. Three 123 researchers were involved in coding and analysis, and sections of data which were doubly 124 coded were compared to increase rigour of analysis and interpretation. Finally, to make the 125 data more "useful" for our programme theory informed approach we mapped out the key 126 inputs, activities, outcomes as a modified logic model. This is a graphic depiction of the 127 shared relationships between the resources, activities, outputs, and outcomes/impacts for a 128 healthcare program (Centres for Disease Control and Prevention, 2018).

\section{Ethics}

130 This study received ethical approval from the Cork Research Ethics Committee (reference: 131 ECM4(h)/ECM3(zzzzz)). The survey landing page provided detailed study information and 132 outlined that by completing and submitting the survey, participants were consenting to take 133 part. 
$134 \quad$ Funding

135 This research was funded by the Health Research Board, who had no role in the research 136 design or conduct.

137

138

139

140

141

142

143

144

145

146

147

148

149

150

151

152

153

154

155

156

157

158

159

160

161

162

163

\section{Results}

\section{Survey respondents}

After deleting cases with significant missing data, the final sample was 112. It wasn't possible to calculate response rate, as some organisations didn't provide the number of subscribers on their mailing lists, and owing to the "snowball" approach. Responding sample details are in Table 2.

\section{$\ll<$ INSERT TABLE 2 ABOUT HERE $\gg>$}

\section{Ranking the features and activities of a model}

Survey participants ranked the importance of general aspects and specific activities of a community dementia palliative care model. Results are shown in figures 1 and 2.

\section{$\ll$ INSERT FIG 1 AND FIG 2 ABOUT HERE $\gg>$}

\section{Qualitative analysis of open-ended question responses}

Most of the data produced by the survey method was rich qualitative data. The four guiding research questions were used to organise the final themes in the data, thus the results are presented as addressing each research question below.

\section{What do stakeholders consider to be the key principles of care that should inform a model?}

Responses merged the philosophies of palliative care and good dementia care, and included domains of the framework for dementia palliative care (Van der Steen, 2014). Respondents emphasised a "holistic" or "palliative care" approach, including the four pillars of palliative care i.e. physical, psychological, social and spiritual care. Equally, many emphasised person-centred care as a core philosophy, i.e. "all care should be person-centred and relevant to the individual's needs". The latter may be summarised as combining individualised care with the aim of optimising quality-of-life. 
164 Some respondents addressed the core philosophies more generally, highlighting the care 165 principles of "empathy" including respect, dignity and compassion. Respecting PwDs' wishes 166 to stay at home was mentioned, as was respecting the families' wishes. Others included 167 end-of-life care specifically:

168 "A model focussed on wellbeing and quality-of-life that is open and honest about what to expect during the end-of-life process for people with dementia" (Occupational Therapist, acute hospital)

\section{What do stakeholders consider to be the most important components/activities of a} model?

Interventions to support "meaningful living". This theme concerned having access to specific interventions targeting the four pillars of palliative care. These activities should be meaningful to the PwD and help to maintain wellbeing. Examples included cognitive stimulation/rehabilitation therapies, reminiscence therapy, social activities, music/art therapies, gardening, and respite facilities in local areas. Wherever possible these should be offered as home-based interventions, with follow-up.

"Access to regular therapies brought to the person in the community. Not for them to travel to the service!" (Clinical Nurse Manager, social care)

Others mentioned social activities e.g. "support for $P w D$ to attend a match, go to the pub, mass or visit a friend". The importance of "regular re-assessment of needs" was highlighted so the PwD is referred to the appropriate treatments, therapies and supports.

Support for the carer was a component considered essential for this community model. Having formal and peer-support networks for carers and families was highlighted to provide emotional and social and practical support. Sitting services, effective respite, 24/7 support, and fast access to services were all critical to "ease carer burden" and support them to care for their loved one at home for as long as possible. Of these factors- increasing home help availability- was considered most critical:

\section{"Appropriately trained home care and funding for it" (Medical Social Worker, acute care)}

\section{"Lack of home care packages- huge barrier to delivering community palliative care"} (Consultant in Geriatric Medicine, acute hospital and hospice) 
195 Importantly, the family should be part of the care team and involved in care decisions. Some 196 respondents felt that this could be improved in the context of palliative care, for example 197 HCPs "do not always listen to family members when they are trying to tell them about pain" 198 (Admiral Nurse, community palliative care).

Information, education, and training was a key theme. It's important that PwD and families are offered education, at an appropriate pace, about the nature of the disease, and what palliative care supports are available should they need them.

Suitably trained and motivated staff are needed. Dementia professionals should all be able to provide generalist palliative care support. Equally, palliative care staff should have training in dementia care, and/or a dementia specialist should be available to palliative care teams or situated in hospices. As one Dementia Specialist Nurse in a hospice setting wrote:

"I think there is lack of understanding on what advanced dementia is and how it presents."

Other related activities were access to education courses, specifically around advanced dementia and end-of-life care in dementia for both families and professionals, and moving and handling training for carers.

212 Information resource packs were suggested so that the PwD or carer can self-refer to 213 services. One respondent suggested having a manned information stand at clinics "for 214 handing out information and whatever the client or family member may need at that time" 215 (Care Home Liaison Nurse, community).

217 Supportive and safe environments which are comfortable and familiar to the PwD was also 218 mentioned, often referring to supporting PwD to live in their homes as long as possible.

219 Having "access to suitable equipment as needed" to allow care at home was considered 220 important, along with "providing equipment as a matter of urgency". Adopting "preventative 221 approaches" e.g. falls prevention was related.

222 Creating wider dementia friendly environments in the community through advocacy, 223 education and "more local community awareness raising" was highlighted. Enabling 224 environments where hospital, community \& primary care providers are supported to provide 225 palliative care were discussed. Supportive environments are important throughout all stages 226 including a "therapeutic environment at preferred place of death". 
228 Symptom management was another common theme; aspects discussed were, management

229 of pain; bowel and bladder; skin care; behaviour; medication; nutrition; swallowing; and 230 prevention and management of delirium; falls. Where possible these should be managed at 231 home, by the carer, supported by community care teams. In advanced disease more 232 expertise from the MDT might be needed, e.g. the palliative management of pain (especially 233 for people who cannot verbally express pain), medication, and controlling symptoms. Access 234 to the MDT within primary care was suggested.

235

Advanced care planning (ACP) was a key activity. ACP should be undertaken early, and reviewed regularly. Several respondents expressed that PwD and their family should be encouraged to engage in ACP following diagnosis; to use this opportunity of contact to discuss the importance of having conversations about the end-of-life stage early in the disease prognosis:

"A plan from diagnosis, where the post-diagnostic team gently start to discuss ACP with the person and family- this can be updated/changed as needed whilst the person still has capacity. This will then influence the care given in the palliative stages!" (Palliative Care Nurse Specialist, hospice and community)

Excellent end-of-life care. To ensure a 'good' death, PwD should be supported to die at their preferred place (e.g. home or hospice), and effective symptom and pain management with an appropriate tool to recognise pain must be provided. Trained HCPs are key "to ensure a respectful and dignified passing". Bereavement support and end-of-life counselling are crucial to support the carer.

251

252 III. How should a model be organised?

253 Comprehensive support. Current services were criticised as "very limited and sporadic".

254 Respondents felt that a broad range of services should be available, so that a

255 comprehensive care plan can be tailored to individual situations, including financial,

256 emotional, physical, social, spiritual and bereavement support, and practical advice. 
257 There should be regular comprehensive needs assessment throughout the PwD's journey.

258 This included receiving regular and standardised palliative care needs assessments, and 259 specialist palliative care (SPC) for patients with complex needs.

260 It's important that services are flexible and responsive to ensure timely identification of, and 261 appropriate responses to, potential crises:

"the model should be flexible so that it can be truly patient-centred" (Admiral Nurse Lead, community)

No one model will be able to support the various needs of individuals at different times of their dementia journey, so a flexible model is key:

"Each individual will need support and advice at different points in their journey." (Admiral Nurse Lead, community)

Integration of services. This involves integrating primary care, community palliative care, mental health, voluntary organisations, etc., with good links to acute services. Excellent communication between these settings is a necessity. Many felt that dementia and palliative care services must be integrated, i.e. that generalist and specialists should work together, for example that a Geriatrician remain primarily involved in care throughout, with support from SPC as required.

Many highlighted the importance of a key worker to "develop a relationship with family and services.

278

"Having a key worker (such as Admiral Nurse) involved start to finish to coordinate care and ensure a palliative care approach is achieved from point of diagnosis through to after death support for families" (Admiral Nurse, hospice)

System level. At the highest level, key elements included that the model is linked to other relevant pathways, is provided as part of 'mainstream' services, that adequate facilities within services are available, that electronic patient data is available to facilitate communication, and that there is a "feedback loop" from service users to enhance the component services. 
Access. Many felt that PwD should have equal access to SPC as people with cancer or any other life-limiting condition.

"People need to be identified as having a chronic disease and to be on the palliative care register so regular monitoring of their care is given" (Admiral Nurse, charity)

Ease of access to all services was also stressed, whether palliative-, dementia-, or care-ofthe-older-person specific, to help PwD to remain at home. Services should be available nationally so a PwD is not limited by their locality, and services should be available free at point of access.

Palliative care consideration from diagnosis. A greater emphasis on addressing the needs of PwD from diagnosis to end-of-life is needed. Not everyone diagnosed with dementia is placed on the palliative care register (where available), which highlights the lack of recognition that dementia is a terminal illness. A "recognition by all that a palliative approach to dementia care is best" is crucial for successful organisation. The need for a clearly defined pathway for dementia aligned to an end-of-life care framework was also expressed:

"There needs to be a clearly defined pathway for dementia aligned to the End-of-Life Care Operational System phases as to who will be involved and lead and how and where handovers should take place on a planned basis" (Assistant Director, primary/community and secondary care)

\section{Who should be involved?}

Multidisciplinary approach. Many professions and disciplines were mentioned as important for a community model of palliative care for dementia, including GPs, community teams, consultant geriatricians, psychogeriatricians, old age psychiatrists, dementia and palliative care nurse specialists, community/public health, psychiatric nurses, psychologists, social workers, therapists, and spiritualists. Furthermore, physiotherapists, dieticians, pharmacists, voluntary groups (e.g. Alzheimer UK or Alzheimer Society of Ireland), volunteers, and local 'grass roots' leaders were also mentioned. Finally, the PwD and carer were included in the MDT by numerous respondents. It is important that the MDT works together and coordinates all inputs to "place as little burden [as possible] on carers and family", while including them in care and care planning. Involving district nursing teams in palliative care approaches is also crucial. Recognising the role of every HCP is important, for example the role that SLTs and 
320 OTs can play in managing communication difficulties and adapting homes quickly and safely 321 was expressed:

322 "SLTS have a huge and largely unrecognised role in managing cognitive communication 323 difficulties and dysphagia, which is an invariable consequence of dementia". (Speech and Language Therapist, private practice)

\section{What are perceived barriers to a model?}

\section{Poor Organisation of Healthcare Systems}

Lack of resources. Respondents discussed many problems at the wider healthcare service level. A lack of funding and resources was mentioned frequently. One respondent felt that there is an "over reliance on voluntary agencies to plug holes in the health system".

331 Dementia palliative care was seen as being costly to provide considering the required home 332 care, 24-hour care, including care for the carer, and rapidly rising numbers of PwD. There's 333 a lack of community palliative care (for any illness), and inadequate home supports including 334 overnight support and on-call GPs. Person-centred care interactions require time, when HCPs are already stretched; currently community care is too focused on completing tasks. There is also a perceived shortage of disciplines such as consultants and nurse specialists, and specialists in dementia with palliative care training, and vice versa. Where good pockets of services existed, this was inequitable and varied by location.

Poor quality of services. Other services may be available, but not fit for purpose, typically existing community services. "No alternative to long-term care" was frequently mentioned as a barrier. Options for respite were poor, as were dementia-friendly leisure activities.

Poor organisation and delivery. Barriers were also identified in how the system is organised. There is an "over-reliance on the medical model", on providing physical care. This is compounded by the poor integration between health and social care:

"Professionals are working in silos with poor communication between disciplines".

(Programme Manager, health agency)

349 Services are difficult to navigate in the context of a complex decision framework. Many PwD 350 aren't assessed for needs until there is a crisis. Hence, there is a need for "a good referral 351 pathway or link to specialist services without having to go through A\&E”. Another problem 
352

353

354

355

356

357

358

359

360

361

362

363

364

365

366

367

368

369

370

371

372

373

374

375

376

377

378

379

380

381

382

was that as nursing homes are viewed as already providing end-of-life palliative care, they are deprioritised for SPC input.

Attitude. Some felt that there is a general lack of interest in society, and among some HCPs, in advancing dementia care as a priority, the following quotes are illustrative:

"[there are] few consultants with a specialist interest in this area." (Consultant in Geriatric Medicine, acute hospital and hospice)

"Specialist palliative care staff feel that this is not where their skills and practice should be directed" (Nurse Consultant End of Life, community)

\section{Misconceptions of Dementia Palliative Care}

Misconceptions about dementia were frequently cited as barriers. Dementia is not being recognised as life-limiting, preventing HCPs from appreciating the benefit of palliative care involvement. It was also felt that there is a general stigma in society (including among HCPs) about dementia, that PwD may be "written off". Related was a fear of non-cognitive symptoms of dementia (i.e. responsive behaviours) stemming from a lack of education. Many HCPs who aren't dementia specialists may not feel confident in caring for people with non-cognitive symptoms manifesting through behaviour.

Misconceptions about palliative care were also pervasive. Not surprisingly, palliative care is "focused mainly on cancer patients", although some, particularly in England, reported that their local SPC service would see PwD. Equating palliative care with end-of-life care or SPC, was common.

"Some healthcare professionals believe that palliative care can only be provided by SPC services" (Clinical Nurse Specialist, community palliative care)

These misconceptions led to access barriers; some hospices won't accept PwD, and other hospices felt unable to manage the longer term needs of PwD.

\section{Delivery of Dementia Palliative Care}

Advance Care Planning. A lack of ACP was a significant and common barrier identified. Too late referrals to SPC impeded excellent end-of-life care, and reactionary interventions 
383 impeded good care throughout the illness. Respondents felt that there is also a "lack of 384 robust training for health professionals" to deal with all stages of dementia and not just 385 diagnosis.

"Much of geriatric training has been in diagnosis and management with little focus on ACP and end-of-life." (Consultant in Geriatric Medicine, acute hospital and hospice)

One respondent recounted her experience of the reluctances of HCPs to engage in early ACP:

$$
\begin{aligned}
& \text { "The main gap in our area is with the post-diagnostic team being reluctant to become } \\
& \text { involved in ACP discussions" (Palliative Care Nurse Specialist, hospice and community) }
\end{aligned}
$$

There appears to be no clear guidance on when and by whom this discussion should take place among HCPs particularly when dealing with early stage dementia.

Dementia specific barriers. Dementia that isn't diagnosed, or delayed diagnosis, impedes effective palliative care. Difficulties in prognostication or staging dementia were acknowledged. Physical needs assessments, including pain, are more difficult in PwD and require specific skills. Some felt that it takes more time to care for PwD, and that they need a higher level of home support.

\section{What would facilitate implementation of a model?}

Funding. Sufficient funding is essential for adequate resources and increase staffing levels, particularly within the community. With adequate funding, the core components of the model can be supported.

406

407 Education and training. This included training in dementia and in palliative care for all HCPs 408 who are interacting with PwD. Specific topics may include communication, including non409 verbal communication, specific training in ACP and end-of-life for both healthcare 410 professionals and carers, reminiscence for PwD and carers, education with carers in the 411 home "on what to expect with palliative illness and dementia", and broader education on 412 living with dementia: 
"Education on what the world is like for someone living/dying with dementia - for healthcare professionals, family, carers and community groups so that individuals in the wider community know how to support someone with dementia" (Dementia Champion, hospice)

Carer support. Any model requires significant unpaid or informal caring from a family member, who may often be older, frail, and/or ill themselves. Respondents mentioned group community supports, courses in caring, and better respite options.

"Respite is critical to keeping people in their own homes especially as the illness progresses." (Consultant Nurse in Palliative Care, acute hospital)

Therapeutic relationships. Respondents felt it essential that staff are friendly, know the PwD and family well, and practice clear, effective communication. This would facilitate personcentred care. The model should strengthen relationships between PwD and their carers as a supportive family is often the key element which enables $\mathrm{PwD}$ to remain in the community.

Other facilitators. A range of practical elements were mentioned as facilitators, these included: transport to community services, assistive technologies, home equipment, reliable assessment and prognostication tools.

\section{Logic Model}

A logic model (figure 3) was developed to illustrate how the emergent themes might be translated to a healthcare model. Key themes were mapped onto the elements of 'input', 'intervention', 'components', 'intermediate and long-term outcomes', and 'contextual and external factors'.

\section{$\ll$ FIGURE 3 ABOUT HERE $\gg>$}

\section{Discussion}

A model of care broadly describes a framework that brings together regulatory, organisational, financial, and clinical aspects of service provision to outline best practice in patient care delivery (NCPPC, 2019). While current practices around dementia palliative care are suboptimal, having clear models will assist healthcare providers in different 
444 countries provide better care and to a higher standard. Although increasingly dementia is 445 being recognised as a terminal illness and palliative care is being increasingly promoted, a 446 challenge remains in defining what works, and/or would work well in real-life practice. This 447 survey addressed this challenge. Responses were collected from stakeholders across five 448 different countries with four healthcare systems and a relative degree of consistency was 449 observed, with key themes emerging. While the initial model will be developed for an Irish 450 context, the current results have wider relevance.

451 The seminal EAPC white paper (Van der Steen, 2014) identifies 11 domains of optimal 452 dementia palliative care, namely: person-centred care, communication and shared decisionmaking; optimal treatment of symptoms and providing comfort; setting care goals and advance planning; continuity of care; psychosocial and spiritual support; family care and involvement; education of the health care team; societal and ethical issues. prognostication and timely recognition of dying; avoiding overly aggressive, burdensome or futile treatment; and applicability of palliative care. All of these domains emerged as themes within our survey, reinforcing their relevance. However, our "bottom-up" qualitative approach highlighted some interesting nuances within these themes. Furthermore, by framing the results in a logic model we highlight what a service-delivery model of dementia palliative care might look like, and the interplay between different model domains or components.

There was a significant degree of consistency in the responses provided by key stakeholders, even across the different regions; these are represented in the modified logic model. The inputs required for the model include funding and resources, and staff education As highlighted in the logic model, contextual and external factors must be considered when and time, however longer-term outcomes may provide a cost saving to the health service, planning the implementation of this model, such as the ageing population and current attitudes towards dementia by the public, politicians, and HCPs. Raising awareness about palliative care and dementia among the general public is important to normalise this connection. Creating awareness and educating about dementia generally will help to develop supportive communities and environments for PwD and their carers, which is important for PwD to remain living at home for longer (Lin, 2017).

Potential core components of the model were identified as person-centred care, comprehensive care, integrated care, accessible care, timely care, and care for carer.

476 However, the different model components are inextricably linked, for example: having rapid access to services supports care at home; earlier access to services promotes earlier ACP 478 and better end-of-life outcomes. The broad components can be aligned with existing 
479 frameworks such as the Irish National Clinical Programme for Palliative Care's 'eight 480 foundations'(NCPPC, 2019), and the 'six ambitions' of the UK's Ambitions for Palliative and 481 End of Life Care framework (2015); both of which were developed as relevant for the 482 palliative care of all adults with a life-threatening illness. However, many issues specific to 483 dementia have been highlighted, hence a specific integrated model for dementia palliative 484 care is crucial (Van der Steen, 2014; Tolson et al., 2017). Our study indicated the perceived 485 importance of components that would perhaps not be part of usual palliative care services, 486 to support meaningful living", such as cognitive stimulation/rehabilitation therapies, 487 reminiscence therapy, etc. The need for supportive and safe environments is also quite 488 dementia specific. The particular challenges of non-cognitive symptoms manifesting through 489 behaviour were also highlighted, along with difficulties in prognostication.

490 Reflecting the above unique needs of a PwD, it is not surprising that providing information, 491 education, and training is integral to the implementation of this model. Others have noted 492 that a skill and discipline mix, alongside an investment in staff development, is required for 493 effective dementia palliative care (Tolson et al., 2017). Even if a SPC service does not 494 provide a dementia palliative care service, as people live longer and dementia in the older 495 population becomes more frequent, more people in the service will have co-morbid 496 dementia. Therefore, it is imperative that SPC professionals upskill in dementia care.

497 Equally, dementia care professionals need to recognise that a palliative care approach can 498 be (and often already is) provided by all disciplines caring for older people and/or those with 499 dementia, and to upskill where appropriate. Critically, these two services must work together 500 within an integrated care model. Further recognition of the value of different non-medical 501 disciplines will also support comprehensive care.

502 Person-centred care is recognised as a core value for dementia care (Kitwood, 2011). This 503 dovetails nicely with the holistic approach of palliative care. To facilitate this, HCPs must 504 communicate effectively with each other whilst including the PwD and their family through 505 regular follow-ups. ACP is a key activity, associated with better end-of-life outcomes for PwD 506 and their carers (Dixon et al., Karagiannidou and Knapp, 2018). All HSCPs can play a role in 507 facilitating ACP (Scott et alet al., 2013).

508 Having a 'key worker' is an important component of the model which can help to keep the 509 lines of communication open between the PwD, their families, and the care team. A support 510 worker role has been shown to improve the quality-of-life of the PwD and the carer, reduce 511 carer burden, improve symptom severity and reduce institutionalisation rates (Goeman et al., 512 Renehan and Koch, 2016). The role is facilitated by ensuring that the key worker/support 513 worker has a skilled background, providing ongoing follow-up (home visits, via phone) that is 
514 based on needs and that the they have the time and ability to develop relationships 515 (Goeman et al., ,Renehan and Koch, 2016).

516 Most PwD live in the community (Pierse et al., O'Shea and Carney, 2019). Support for family 517 members and carers to provide care is essential to a community-based model. Effective 518 respite options (O'Shea et al., 2017), and community and out-of-hours support services are 519 needed to support the PwD to remain at home (Kim et al., 2012). This support needs to be 520 available and offered to carers regularly regardless of their health status, as any carer is 521 prone to experience burnout which may result in the carer feeling that they can no longer 522 care for the PwD at home (Donnelly et al., 2015).

523 Another key strength of the current draft model is that it considers what aspects of palliative 524 care may be delivered to PwD at any disease stage; many frameworks focus on palliative 525 care for advanced dementia only (Tolson et al., 2016). Aspects like approaching ACP early 526 in the disease and having a skilled dementia key worker who can link into palliative care 527 supports early if needed, extend the remit of palliative care to a whole disease focus.

528 For any such model to be successful, its implementation must be carefully considered 529 (Mooreer et alet al., 2015). Tolson et alet al (2016) discuss the Prudent Healthcare 530 Paradigm- the delicate balancing act between effective and efficient policy making and policy 531 implementation in relation to advanced dementia. This paradigm acknowledges the 532 economic pressures and inequalities in provision which mean that existing care systems 533 cannot simply expand to meet the challenge of their ageing populations. Instead 534 transformational change is required in which everyone takes responsibility for health and 535 wellbeing in a united and integrated way. In many countries the context for such a change is 536 positive. In Ireland, and other European countries, there is a rapidly growing recognition by 537 governments, healthcare systems, and policy groups of the importance of palliative care for 538 non-cancer illnesses including dementia. Ireland has a National Dementia Office with a specific remit to implement the National Dementia Strategy,_and of note the strategy,

540 perhaps reflecting its relatively later development (in 2014) than strategies in other European 541 countries, has a very strong focus on palliative care. However close working with the Irish

542 National Clinical Programme for Palliative Care would be critical to successful 543 implementation.

544 Some limitations of the current study should be noted. The survey was modest in size with $545 \mathrm{n}=112$ respondents across five countries. Positively, distribution was targeted so that 546 respondents represented key informed stakeholders, however no claims to 547 representativeness can be made. Furthermore, the components represented in the logic 548 model are tentative, and the final model will need to be informed by further research, 
549 including site visits and evaluations and further in-depth stakeholder consultation to include 550 PwD. These components however shed light onto what the potential model might look like, 551 and are a useful starting point to inform further research.

\section{Conclusion}

553 Utilising a palliative care approach can improve the outcomes for PwD and their carers, 554 especially when a PwD's illness progresses to advanced dementia. Such outcomes may 555 include improved quality-of-life for both the PwD and their family, improved quality-of-care, 556 better clinical outcomes, more PwD dying in their preferred place, improved patient

557 experiences, cost saving to the health service, and PwD being treated with respect, dignity 558 and compassion. While the relevance of a palliative care approach for PwD is now widely 559 accepted, the challenge has remained in how to achieve this in practice. The results of this 560 study address this by informing a wider research project to develop a service-delivery model, 561 initially for Ireland, but with potential for adaptation by other national health services, or other 562 key groups who provide dementia and or palliative care services.. 


\section{References}

565 Braun, V. and Clarke, V. (2006) Using thematic analysis in psychology. Qualitative research in

566 psychology, 3(2), pp.77-101.

567 Brodaty, H., Seeher, K., \& Gibson, L. (2012) Dementia time to death: a systematic literature review

568 on survival time and years of life lost in people with dementia. International Psychogeriatrics, 24(7),

569 1034-1045. doi: 10.1017/S1041610211002924

570 Centres for Disease Control and Prevention. (2018) Program Evaluation Framework Checklist for Step

571 2: Describe the Program. Retrieved 28 April, 2020, from

572 https://www.cdc.gov/eval/steps/step2/index.htm

573 Dempsey, L., Dowling, M., Larkin, P. and Murphy, K. (2015) 'The unmet palliative care needs of those

574 dying with dementia', International Journal of Palliative Nursing, 21(3), pp.126-133.

575 Dening, K.H. (2016) 'Palliative and end-of-life care for people with dementia', Nursing Standard

576 (2014+), 30(23), pp.42.

577 Dening, K.H., Sampson, E.L. and De Vries, K. (2019) 'Advance care planning in dementia:

578 recommendations for healthcare professionals', Palliative care, 12.

579 Dixon, J., Karagiannidou, M. and Knapp, M. (2018) 'The Effectiveness of Advance Care Planning in 580 Improving End-of-Life Outcomes for People With Dementia and Their Carers: A Systematic Review 581 and Critical Discussion', Journal Of Pain And Symptom Management, 55(1), pp.132-150.e1.

582 Donnelly, N.A., Hickey, A., Burns, A., Murphy, P. and Doyle, F. (2015) 'Systematic review and meta583 analysis of the impact of carer stress on subsequent institutionalisation of community-dwelling older 584 people', PloS one, 10(6).

585 EU Joint Programme-Neurodegenerative Disease Research (2014) Palliative and end-of-life care 586 research in neurodegenerative disease. Report of the JPND Action Group. Available at:

587 http://www.neurodegenerationresearch.eu/initiatives/jpnd-alignment-actions/palliative-care/

588 Fox, S., Cahill, S., Foley, M., Lynch, M. and Timmons, S. (2017) Demonstrating the Potential Benefit of 589 Palliative Care Input for People with Dementia, Using Case Studies. Age and Ageing, 46(Suppl_3). 
590 Goeman, D., Renehan, E. and Koch, S. (2016) 'What is the effectiveness of the support worker role

591 for people with dementia and their carers? A systematic review', BMC health services research, 592 16(1), pp.285.

593 Kim, H., Chang, M., Rose, K. and Kim, S. (2012) 'Predictors of caregiver burden in caregivers of 594 individuals with dementia', Journal of advanced nursing, 68(4), pp.846-855.

595 Kitwood, T. (2011) 'Dementia reconsidered: the person comes first', Adult lives: A life course 596 perspective, 89.

597 Lin, S.Y. (2017) 'Dementia-friendly communities' and being dementia friendly in healthcare settings', 598 Current opinion in psychiatry, 30(2), pp.145.

599 Moore, G.F., Audrey, S., Barker, M., Bond, L., Bonell, C., Hardeman, W., Moore, L., O'Cathain, A., 600 Tinati, T., Wight, D., Baird, J. (2015) Process evaluation of complex interventions: Medical Research 601 Council guidance. BMJ. 350:1258.

602 Nakanishi, M., Nakashima, T., Shindo, Y., Miyamoto, Y., Gove, D., Radbruch, L., \& Van der Steen, J. 603 (2015). An evaluation of palliative care contents in national dementia strategies in reference to the 604 European Association for Palliative Care white paper. International Psychogeriatrics, 27(9), 1551605 1561. doi:10.1017/S1041610215000150

606 National Clinical Programme for Palliative Care (2019) Adult Palliative Care Services, Model of Care 607 for Ireland. Health Service Executive.

608 National Palliative and End of Life Care Partnership (2015) Ambitions for Palliative and End of Life 609 Care: A national framework for local action 2015-2020. National Palliative and End of Life Care 610 Partnership, UK.

611 O'Shea, E. and Monaghan, C. (2017) 'An economic analysis of a community-based model for 612 dementia care in Ireland: a balance of care approach', International psychogeriatrics, 29(7), pp.11756131184.

614 O'Shea, E., Timmons, S., O'Shea, E., Fox, S. and Irving, K. (2017) 'Key stakeholders' experiences of 615 respite services for people with dementia and their perspectives on respite service development: a 616 qualitative systematic review', BMC geriatrics, 17(1), pp.282.

617 Palinkas, L. A., Horwitz, S. M., Green, C. A., Wisdom, J. P., Duan, N., \& Hoagwood, K. (2015).

618 Purposeful Sampling for Qualitative Data Collection and Analysis in Mixed Method Implementation 
619 Research. Administration and policy in mental health, 42(5), 533-544. doi: 10.1007/s10488-013-

$620 \quad 0528-y$

621 Pierse, T., O'Shea, E. and Carney, P. (2019) 'Estimates of the prevalence, incidence and severity of 622 dementia in Ireland', Irish journal of psychological medicine, 36(2), pp.129-137.

623 Rhodius-Meester, H. F. M., Tijms, B. M., Lemstra, A. W., Prins, N. D., Pijnenburg, Y. A. L., Bouwman, 624 F., . . van der Flier, W. M. (2019) Survival in memory clinic cohort is short, even in young-onset 625 dementia. Journal of Neurology, Neurosurgery \&amp;amp; Psychiatry, 90(6), 726. doi: 10.1136/jnnp$626 \quad 2018-318820$

627 Scott, I.A., Mitchell, G.K., J Reymond, E. and Daly, M.P. (2013) 'Difficult but necessary 628 conversations - the case for advance care planning', Medical Journal of Australia, 199(10), pp.662629666.

630 Tolson, D., Holmerova, I., Macrae, R., Waugh, A., Hvalič-Touzery, S., de Abreu, W., . . Hanson, E. 631 (2017) Improving Advanced Dementia Care: An Interprofessional Palliare Learning Framework. 632 Journal of the American Medical Directors Association, 18(7), 561-563. doi:

$633 \quad 10.1016 /$ j.jamda.2017.03.014

634 Tolson, D., Fleming, A., Hanson, E., de Abreu, W., Crespo, M. L., Macrae, R., . . Routasalo, P. (2016)

635 Achieving prudent dementia care (palliare): an international policy and practice imperative.

636 International journal of integrated care, 16(4).

637 Van der Steen, J.T., Radbruch, L., Hertogh, C.M., de Boer, M.E., Hughes, J.C., Larkin, P., Francke, A.L., 638 Jünger, S., Gove, D., Firth, P. and Koopmans, R.T. (2014) White paper defining optimal palliative care 639 in older people with dementia: a Delphi study and recommendations from the European Association 640 for Palliative Care. Palliative medicine, 28(3), pp.197-209.

641 World Health Organisation (2019) Dementia: Key Facts. Available at: https://www.who.int/news$642 \mathrm{room} / \mathrm{fact}-\mathrm{sheets} /$ detail/dementia (Accessed: 12/Feb/2020). 
Table 1. Survey question items

\begin{tabular}{|c|c|}
\hline \multicolumn{2}{|c|}{ Open-ended items } \\
\hline Question 1 & $\begin{array}{l}\text { What do you think a good model of palliative care for people with dementia in the } \\
\text { community looks like? }\end{array}$ \\
\hline Question 2 & What principles of care should inform such a model? \\
\hline Question 3 & $\begin{array}{l}\text { In your opinion who are the key people/professionals who should be involved in a } \\
\text { model for palliative care for people with dementia in the community? }\end{array}$ \\
\hline Question 4 & $\begin{array}{l}\text { In your opinion, what are the key activities (e.g. component, intervention, other) } \\
\text { that should be included in a model for palliative care for people with dementia in } \\
\text { the community? }\end{array}$ \\
\hline Question 5 & Do you have any other comments relating to the above questions? \\
\hline Question 6 & $\begin{array}{l}\text { Please list any current barriers that you can think of to providing palliative care to } \\
\text { people with dementia living in the community. }\end{array}$ \\
\hline Question 7 & Please comment on any ways in which these barriers might be overcome. \\
\hline Question 8 & $\begin{array}{l}\text { We would like to hear about examples of good practice. Please provide any } \\
\text { example, from your experience, of what has worked well in the past for providing } \\
\text { palliative care to people with dementia. This can be a model, component, a way } \\
\text { of working, or other. }\end{array}$ \\
\hline \multicolumn{2}{|c|}{ Closed-ended items } \\
\hline Question 9 & $\begin{array}{l}\text { Below we have listed some general aspects of a good model of palliative care for } \\
\text { people with dementia. Please tell us how important you think each aspect is, by } \\
\text { choosing from Not very important, Somewhat important, Very important, or } \\
\text { Essential. } \\
\text { Enhanced access } \\
\text { Standardised assessment tools } \\
\text { Information continuity } \\
\text { Incorporation of existing guidelines on pharmacological and non- } \\
\text { pharmacological } \\
\text { Treatments } \\
\text { Defined pathways for specialist service input } \\
\text { Mechanisms to measure user experience } \\
\text { Care for people at all stages of illness (i.e. early to advanced dementia) } \\
\text { Based on a theoretical framework } \\
\text { Evidence-based } \\
\text { Other (please specify) }\end{array}$ \\
\hline Question 10 & $\begin{array}{l}\text { Below we have listed some specific components of a good ser } \\
\text { care for people with dementia. Please tell us how important yc } \\
\text { component is, by choosing from Not very important, Somewho } \\
\text { important, or Essential. } \\
\text { Care planning } \\
\text { Case management } \\
\text { Advance care planning } \\
\text { Advocacy } \\
\text { Patient and carer support } \\
\text { Support during transitions of care } \\
\text { Information resources } \\
\text { Directories of local services } \\
\text { Other (please specify) }\end{array}$ \\
\hline
\end{tabular}


Table 2. Respondents' Demographic Data $(\mathrm{N}=112)$

\begin{tabular}{llllll}
\hline (Primary) discipline & N & Country/Region & N & Gender & N \\
\hline Nursing & 50 & England & 43 & Female & 79 \\
Community \& Social Care & 15 & Republic of Ireland & 25 & Male & 13 \\
Doctor & 11 & Northern Ireland & 22 & Missing & 20 \\
Academia & 8 & Wales & 5 & & \\
Physio, OT, SLT, Psychologist & 8 & Scotland & 2 & & \\
Other & 7 & Missing & 15 & & \\
Missing & 13 & & & & \\
\hline
\end{tabular}


Fig 1: Ranking of general aspects of a good model of palliative care for people with dementia.

Standardised assessment tools Based on a theoretical framework

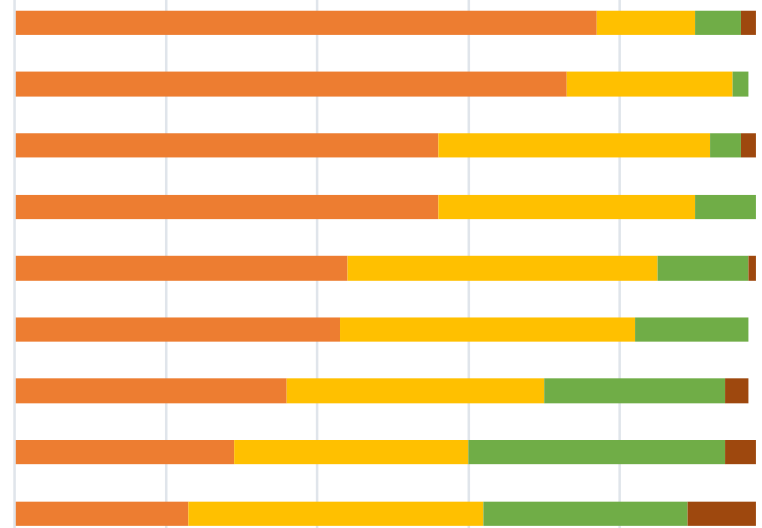

40

60

80 100 
Fig 2: Ranking of specific components of a good model of palliative care for people with dementia.

\section{Patient and care support} Support during transition of care Care planning Advance care planning Case management Information resources Advocacy Directories of local services

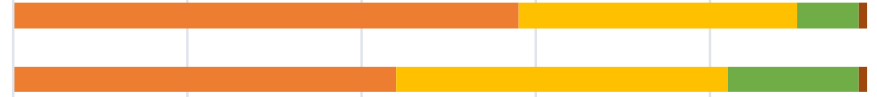

40

60

80 100

Somewhat important

- Not very important 
- Funding

- Resources

- Staff

Education

- Time

Keeps people with dementia at home for as long as possible Is responsive to needs Is individualised

\section{Person Centered care}

- Co-design

- Patient passport

- Advance Care Planning

\section{Comprehensive care}

- 4 pillars of palliative care

- Non-medical and alternative therapies

- Good symptom management

\section{Integrated care}

- Dementia and Palliative Care working together

- Excellent Community team

- Key worker

\section{Accessible care}

- More, flexible, home help hours

- Equitable access to palliative care

Timely care

- Responsive

\section{Care for Carer}

- Involved in decision making

- Individualised \& group supports

- Effective respite

- Bereavement care

\section{Person Centered care}

- Increased understanding and recognition of $P W D$ needs

- Stronger relationships between PwD and the care team

\section{Comprehensive care}

- PwD have full needs assessment

- Palliative care approach applied by all HCPs

- Care Pathway

Integrated care

- Excellent interdisciplinary communication

\section{Accessible care}

- 24/7 access to care

- Home care packages

\section{Timely care}

- Crisis admissions avoided

\section{Care for Carer}

- Increased family engagement

- Family better able to cope at home
- Improved quality of life for PwD, and their families.

- Improved quality of care, including better clinical outcomes, lower mortality

- PwD care for and die in preferred place of care

- PwD treated with respect, dignity, and compassion

- Improved patient experiences

- Cost saving to HSE

${ }_{38}^{37}$ Contextual and External Factors: Ageing population; Public attitudes; Political support; HCPs perception of dementia as a priority; Dementia recognised as a life- limiting illness; Wider emphasis on bio-psycho-social model, De-stigmatising palliative care; HCP 'fear' of dementia. 